\title{
Pressure-induced hydrogen bond symmetrization in iron oxyhydroxide
}

Weiming M. Xu, Eran Greenberg, Gregory Kh. Rozenberg, Moshe P. Pasternak, Elena Bykova, Tiziana Boffa-Ballaran, Leonid Dubrovinsky, Vitali Prakapenka, Michael Hanfland,

Olga Vekilova, Sergey Simak, Igor Abrikosov and R. Ruffer

\section{Linköping University Post Print}

\section{Tweet}

N.B.: When citing this work, cite the original article.

Original Publication:

Weiming M. Xu, Eran Greenberg, Gregory Kh. Rozenberg, Moshe P. Pasternak, Elena Bykova, Tiziana Boffa-Ballaran, Leonid Dubrovinsky, Vitali Prakapenka, Michael Hanfland, Olga Vekilova, Sergey Simak, Igor Abrikosov and R. Ruffer, Pressure-induced hydrogen bond symmetrization in iron oxyhydroxide, 2013, Physical Review Letters, (111), 17.

http://dx.doi.org/10.1103/PhysRevLett.111.175501

Copyright: American Physical Society

Postprint available at: Linköping University Electronic Press

http://urn.kb.se/resolve?urn=urn:nbn:se:liu:diva-97382 


\title{
Pressure-Induced Hydrogen Bond Symmetrization in Iron Oxyhydroxide
}

\author{
Weiming Xu, ${ }^{1}$ Eran Greenberg, ${ }^{1}$ Gregory Kh. Rozenberg, ${ }^{1}$ Moshe P. Pasternak, ${ }^{1}$ \\ Elena Bykova, ${ }^{2}$ Tiziana Boffa-Ballaran, ${ }^{2}$ Leonid Dubrovinsky, ${ }^{2, *}$ Vitali Prakapenka, ${ }^{3}$ Michael Hanfland, ${ }^{4}$ \\ Olga Yu. Vekilova, ${ }^{5}$ Sergei I. Simak, ${ }^{5}$ and Igor A. Abrikosov ${ }^{5}$ \\ ${ }^{1}$ School of Physics and Astronomy, Tel Aviv University, 69978, Tel Aviv, Israel \\ ${ }^{2}$ Bayerisches Geoinstitut, University of Bayreuth, Bayreuth 95440, Germany \\ ${ }^{3}$ Consortium for Advanced Radiation Sources, University of Chicago, 9700 South Cass Avenue, Argonne, Illinois 60439, USA \\ ${ }^{4}$ European Synchrotron Radiation Facility (ESRF), 6 rue Jules Horowitz, BP 220, F-38043 Grenoble Cedex, France \\ ${ }^{5}$ Department of Physics, Chemistry and Biology (IFM), Linköping University, SE-581 83 Linköping, Sweden
}

(Received 29 May 2013; published 24 October 2013)

\begin{abstract}
Under high pressures the hydrogen bonds were predicted to transform from a highly asymmetric soft $\mathrm{O}-\mathrm{H} \cdots \mathrm{O}$ to a symmetric rigid configuration in which the proton lies midway between the two oxygen atoms. Despite four decades of research on hydroxyl containing compounds, pressure induced hydrogen bond symmetrization remains elusive. Following single crystal x-ray diffraction, Mössbauer and Raman spectroscopy measurements supported by ab initio calculations, we report the H-bonds symmetrization in iron oxyhydroxide, $\mathrm{FeOOH}$, resulting from the $\mathrm{Fe}^{3+}$ high-to-low spin crossover at above $45 \mathrm{GPa}$.
\end{abstract}

DOI: 10.1103/PhysRevLett.111.175501

PACS numbers: 61.50.Ks, 62.50.- $\mathrm{p}, 64.60 .-\mathrm{i}, 71.30 .+\mathrm{h}$

The hydrogen bond in oxyhydroxides and hydroxides is an attractive interaction between a hydrogen atom from a hydroxyl $(\mathrm{O}-\mathrm{H})$ group and a near neighbor oxygen atom or a group of atoms [1]. In contrast to other interacting atoms, $\mathrm{H}$ bonds undergo large variations of their energetic and geometrical parameters under pressure [2-5]. At ambient pressure the $\mathrm{O}-\mathrm{H} \cdots \mathrm{O}$ configuration is highly asymmetric. For example, in goethite, $\alpha$-FeOOH, a common mineral in soils, sediments, and ore deposits, the angle formed by the $(\mathrm{O}-\mathrm{H})$ and the adjacent $\mathrm{O}$ is $161(3)^{\circ}$, the $\mathrm{O}-\mathrm{H}$ length is $0.88(4) \AA$, and $\mathrm{H} \cdots \mathrm{O}$ is $1.90(4) \AA[6]$. As observed by Holzapfel [2] even moderate compression reduces the $\mathrm{H} \cdots \mathrm{O}$ distances, barely affecting the hydroxyl bond length, which leads to the strengthening of the hydrogen bond at high pressure [5]. Benoit et al. [3] and Lin et al. [4] predicted that higher compression will also lead to linearization of the $\mathrm{O}-\mathrm{H} \cdots \mathrm{O}$ bond and eventually the formation of a symmetric $\mathrm{O}-\mathrm{H}-\mathrm{O}$ hydrogen species of equal $\mathrm{O}-\mathrm{H}$ distances.

Symmetrization of hydrogen bonds is expected to have a significant effect on crystal structure and the behavior of materials [5]. Suggestions that the phenomena may occur under compression were reported for a number of compounds, namely, the hydrogen halides [7], $\delta$ - $\mathrm{AlOOH}(\mathrm{D})$, $\mathrm{MgSi}_{2} \mathrm{O}_{4}(\mathrm{OH})_{2}, \delta-\mathrm{Al}(\mathrm{OH})_{3}, \mathrm{CrOOH}(\mathrm{D}), \mathrm{GaOOH}, \mathrm{InOOH}$, and formic acid [8-12], but only in $\mathrm{H}_{2} \mathrm{O}$ ice- $X$, at pressures approaching $100 \mathrm{GPa}$, has the elusive symmetrization been unambiguously demonstrated [13-16].

Studies of hydrogen bond properties at pressures exceeding $20 \mathrm{GPa}$ are difficult. The reason is that the ordinary direct and indirect structural methods such as powder x-ray diffraction (XRD), and vibration spectroscopy, Raman or infrared (IR), are unable to locate the position of hydrogen [9-15]. Neutron diffraction, the perfect method for this purpose, is currently limited to pressures of $<20 \mathrm{GPa}$ [11]. Yet the combination of single crystal XRD and vibrational spectroscopy, as demonstrated in the example of ice- $X$ [16], may be adequate to resolve the geometry of the hydrogen bond as a function of pressure. Recent developments of single crystal XRD in diamond anvils cells enable the refinement of atomic positions in complex crystal structures into the $100 \mathrm{GPa}$ range [17]. This creates a unique opportunity to trace the geometry of the hydrogen bond by its effect on the shape of atomic groups or polyhedrons forming crystal structure. For example, the structure of $\alpha-\mathrm{FeOOH}$ (space group Pnma) [6] at ambient conditions consists of highly distorted $\mathrm{FeO}_{6}$ octahedra (Fig. 1) with three short [1.933(3), 1.962(2) A] and three long [2.107(3) $\mathrm{A}] \mathrm{Fe}-\mathrm{O}$ bonds. The longer Fe-O bonds designated as Fe-O1 correspond to the covalently bound oxygen of the hydroxyl, while the other oxygen $\mathrm{O} 2$ ligands are weakly bound to $\mathrm{H}$ of the nearest neighbor hydroxyl. Thus, the octahedral moiety can be described as $\mathrm{Fe}(\mathrm{O} \cdots \mathrm{H})_{3}(\mathrm{O}-\mathrm{H})_{3}$. If, under compression, the hydrogen bonds symmetrize, namely, all $\mathrm{O}-\mathrm{H}$ bond lengths become equal, this should lead to a reduction in the distortion of the $\mathrm{FeO}_{6}$ octahedra easily measurable by single crystal $\mathrm{XRD}$. Thus, a detection of this abrupt reduction of the $\mathrm{FeO}_{6}$ octahedral asymmetry becomes the primary goal of this study.

High pressure structural studies of goethite up to $\sim 29 \mathrm{GPa}$ have been performed by several groups $[18,19]$. Nagai et al. [18] carried out powder XRD studies to $24 \mathrm{GPa}$ and derived a bulk modulus $K_{0}=111(2) \mathrm{GPa}$ and its pressure derivative $K^{\prime}=4$. Studies to $29 \mathrm{GPa}$ by Gleason et al. [19] deduced values of $K_{0}$ and $K^{\prime}$ of 140(4) and 4.6(4) $\mathrm{GPa}$, respectively. The behavior of goethite under pressure has been studied theoretically by Otte 


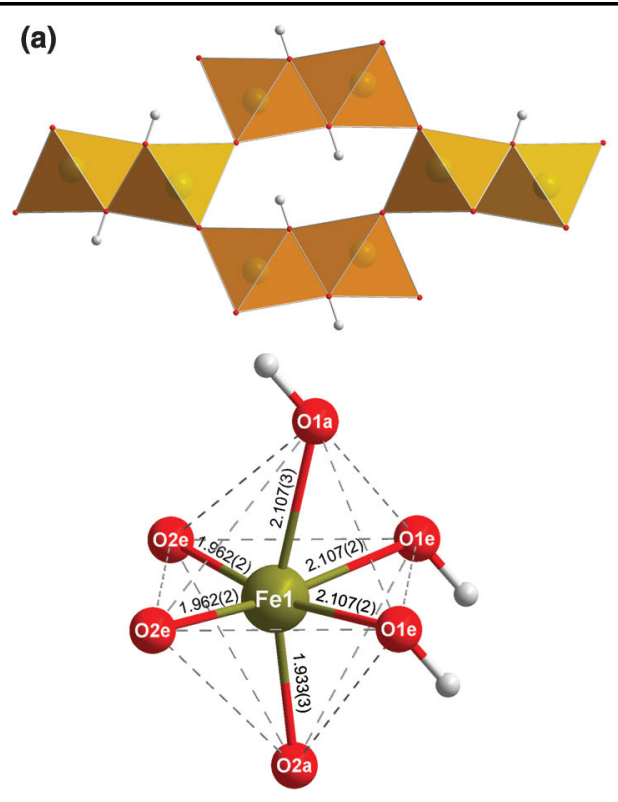

(b)

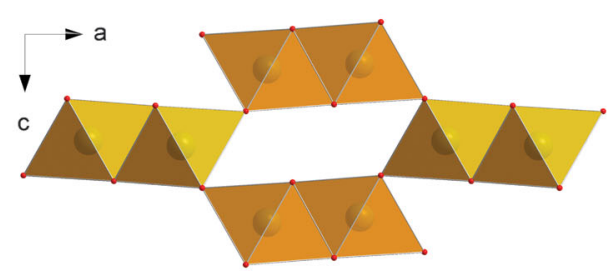

FIG. 1 (color). Crystal structure of $\alpha$-FeOOH along the $b$ direction as well as $\mathrm{FeO}_{6} \mathrm{H}_{3}$ octahedra at ambient pressure (a) and at 47.6(2) GPa (b). $\alpha$-FeOOH crystallizes in an orthorhombic structure (space group Pnma) and can be described in terms of a slightly distorted hexagonally close packed arrangement of $\mathrm{O}$ anions with $\mathrm{Fe}$ cations occupying two-thirds of the octahedral sites. The FeO 6 octahedra are linked together by sharing edges and vertices to form infinite $2 \times 1$ channels parallel to the $b$ axis with $\mathrm{H}$ atoms inside the channels. There are two independent oxygen sites: $\mathrm{O} 1$, at ambient conditions covalently bound to hydrogen (O-H bond), and $\mathrm{O} 2$, characterized by a weak $\mathrm{H} \cdots \mathrm{O}$ bond; in the selected octahedron one can distinguish $\mathrm{O} 1_{a}$ and $\mathrm{O} 2_{a}$ atoms in the axial position and two $\mathrm{O}_{e}$ and two $\mathrm{O} 2_{e}$ atoms in the equatorial position. The octahedral $\mathrm{Fe}(\mathrm{O}-\mathrm{H})_{3}(\mathrm{O} \cdots \mathrm{H})_{3}$ moiety is highly distorted at ambient pressure (a): the Fe-O1 bonds are significantly longer than those of $\mathrm{Fe}-\mathrm{O} 2$, and becomes regular above the transition pressure (b).

et al. [20] using density-functional theory (DFT) which has predicted a pressure-induced high-spin to low-spin (HS-LS) transition at $\sim 8 \mathrm{GPa}$ concurrent with a structural transition to the $\varepsilon-\mathrm{FeOOH}$ polymorph. But taking into account electronic correlation effects at the DFT $+U$ level, the spin crossover in the $\varepsilon$ phase has been shown to take place at $\sim 56 \mathrm{GPa}$. At the same time, a possibility of the HS-LS transition in the $\alpha$ phase has not been discussed in this work. Based on the spin-polarized $a b$ initio calculations within the DFT $+U$ method, Tunega [21] concluded that the antiferromagnetic high-spin (HS) state should be stable up to $80 \mathrm{GPa}$. Because of this, the structural parameters were investigated for the $\alpha-\mathrm{FeOOH}$ polymorph in the HS state only. On the other hand, conventional spin-polarized calculations using the generalized-gradient approximation suggest a HS-LS transition at $\sim 55 \mathrm{GPa}$, and, moreover, DFT $+U$ total energies indicated a possibility of the LS state stabilization at high pressure. In view of the controversies in the structural and electronic properties of the above mentioned studies, diverse experimental structural and electronic methods have been employed in the present work: single crystal and powder XRD, the Mössbauer spectroscopy at variable $(P, T)$, Raman spectroscopy, and resistance $R(P, T)$ studies [22]. The experiment has been supported by state-ofthe-art theoretical calculations [22]. This methodology combination ultimately sheds new light on exciting pressure-induced structural and electronic properties in this transition-metal oxyhydroxide.

Upon compression to $44 \mathrm{GPa}$, the lattice parameters and unit cell volume of $\alpha$-FeOOH gradually decrease (Fig. 2, Fig. S1 in Ref. [22]). A close examination of the dependence of the normalized stress versus the Eulerian strain, $P_{N}\left(f_{E}\right)$ (Ref. [22], Fig. S5), and $V(P)$ shows that at $P>$ $16 \mathrm{GPa}$ the elastic properties change. The best fitting for $V(P)$ could be obtained assuming two different equations of state for the 0-16 and the 16-44 GPa pressure ranges (Fig. 2, see also Ref. [22]). These changes at $\sim 16 \mathrm{GPa}$ are even more obvious from the analysis of interatomic distances. Up to $16 \mathrm{GPa}$ the $\mathrm{O} 1 \cdots \mathrm{O} 2$ distances, involved in the hydrogen bond and located across channels formed by octahedra (Fig. 1), decrease much more rapidly than the Fe-O distances (Fig. 2, Table S1 in Ref. [22]). This indicates that up to $16 \mathrm{GPa}$, similar to isostructural $\alpha$-AlOOH [23], bulk compression takes place through contraction of the channels, involving shortening of the hydrogen bonds, rather than slimming down of the $\mathrm{FeO}_{6}$ octahedra [19]. At $\sim 16 \mathrm{GPa}$ the compressibility of the $\mathrm{O} 1-\mathrm{H} \cdots \mathrm{O} 2$ bond decreases sharply and becomes comparable to the compressibility of the Fe-O bonds (Fig. 2). It is noteworthy, that up to $44 \mathrm{GPa}$ the difference in compressibility of $\mathrm{Fe}-\mathrm{O} 1$ and $\mathrm{Fe}-\mathrm{O} 2$ bonds $(\sim 0.0035 \AA \mathrm{APa}$ for $\mathrm{Fe}-\mathrm{O} 1$ and $\sim 0.0017 \AA / \mathrm{GPa}$ for $\mathrm{Fe}-\mathrm{O} 2)$ affects the shape of the octahedral $\mathrm{Fe}(\mathrm{O} \cdots \mathrm{H})_{3}(\mathrm{O}-\mathrm{H})_{3}$ moiety. The octahedral 


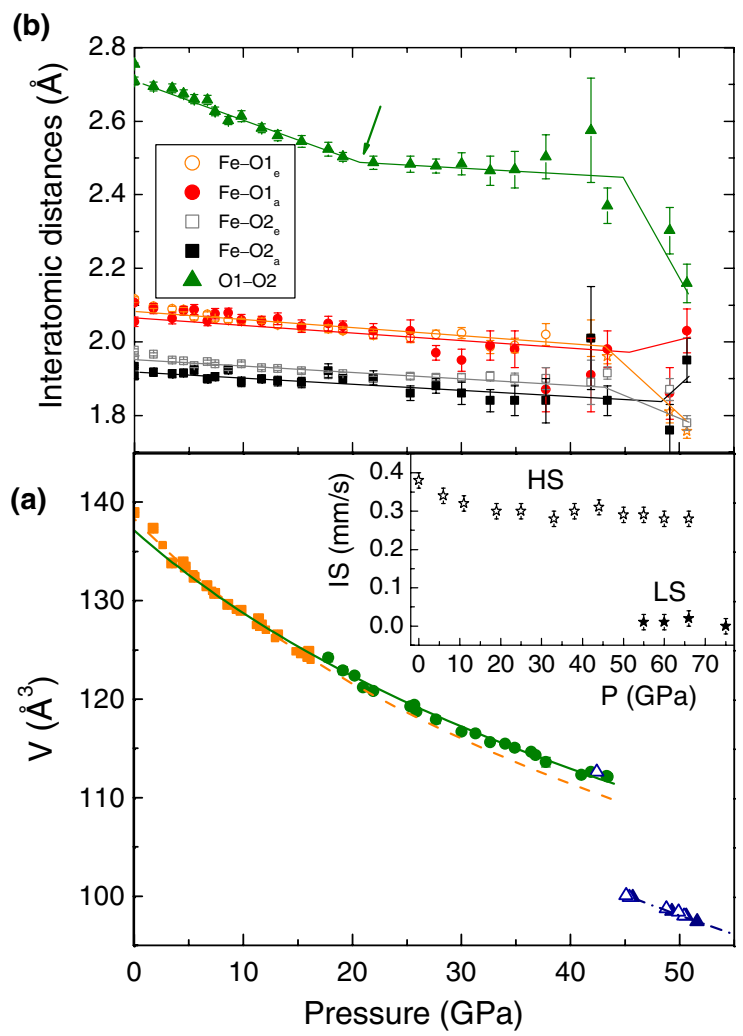

FIG. 2 (color online). Dependence on pressure of unit cell volume (a) and interatomic distances (b) as revealed by in situ $\mathrm{x}$-ray diffraction. The data (a) are divided into three intervals (below $16 \mathrm{GPa}$-orange square symbols, between 16 and $44 \mathrm{GPa}$ - green circles, and above $44 \mathrm{GPa}$ - solid triangles upon compression and open upon decompression), and fitted by 2 nd order Birch-Murnaghan equations of state (curves, dashed orange with parameters $K_{0}=120(3) \mathrm{GPa}, V_{0}=$ 138.4(3) $\AA^{3} /$ unitcell, solid green with parameters $K_{0}=$ 140(5) GPa, $V_{0}=137.2(6)(2) \AA^{3} /$ unit cell, and dash-dot blue $K_{44}=224(13) \mathrm{GPa}, V_{44}=100.5(1) \AA^{3} /$ unit cell; $\left.K^{\prime}=4\right)$. The solid curves (b) serve only as guides to the eyes. The arrow marks a change in pressure dependency of the O1-O2 distances at $\sim 20 \mathrm{GPa}$. The $\mathrm{O}-\mathrm{O}$ and $\mathrm{Fe}-\mathrm{O}$ distances drastically change above $45 \mathrm{GPa}$. The inset (a) shows the pressure dependence of the isomer shift obtained from Mössbauer spectroscopy. Above $50 \mathrm{GPa}$ an onset of the low spin state is observed characterized by an abrupt drop in the IS value, signaling the sharp decrease of the average $\langle\mathrm{Fe}-\mathrm{O}\rangle$ distances.

distortion can be characterized by the deviation $\Delta$ of the $\mathrm{O} 1_{a}-\mathrm{Fe}-\mathrm{O} 2_{a}$ angle from $180^{\circ}$ and by the ratio of the average iron-oxygen distances $\langle\mathrm{Fe}-\mathrm{O} 2\rangle /\langle\mathrm{Fe}-\mathrm{O} 1\rangle$. Figure 3 shows that under compression $\mathrm{FeO}_{6}$ octahedra demonstrate a clear trend to symmetrization, which is nevertheless far from complete at $\sim 44 \mathrm{GPa}$.

At $\sim 45 \mathrm{GPa}$ an isostructural phase transition takes place manifested by a drastic reduction of the molar volume (by $\sim 11 \%$ ), and vanishing of the Raman modes (Fig. 2, Figs. S1, S2 in Ref. [22]). Within the experimental errors the transition is reversible upon decompression with no obvious signs of hysteresis (Fig. 2). The discontinuous

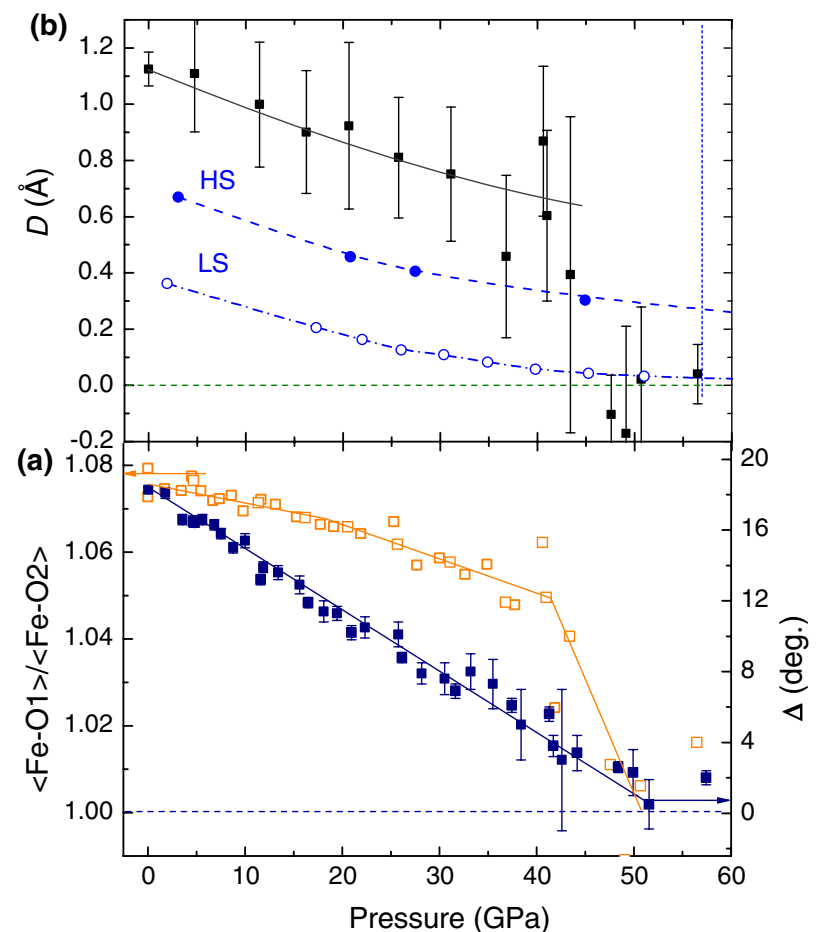

FIG. 3 (color online). Distortion of $\mathrm{FeO}_{6}$ octahedra (a) and asymmetry of hydrogen bond (b) in $\mathrm{FeOOH}$ as a function of pressure. In goethite, distortion of the octahedra (a) can be characterized by the deviation $\Delta$ from $180^{\circ}$ of the $\mathrm{O} 1_{a}-\mathrm{Fe}-\mathrm{O} 2_{a}$ angle (solid squares) or by the ratio of average iron-oxygen distances $\langle\mathrm{Fe}-\mathrm{O} 2\rangle /\langle\mathrm{Fe}-\mathrm{O} 1\rangle$ (open squares) (see Fig. 1 for atoms assignment). The asymmetry of the hydrogen bond $\boldsymbol{D}(\mathrm{b})$ is characterized by the difference in length between $\mathrm{O} 1-\mathrm{H}$ and $\mathrm{O} 2 \cdots \mathrm{H}$. The individual $\mathrm{O} 1-\mathrm{H}$ and $\mathrm{O} 2 \cdots \mathrm{H}$ bonds lengths calculated either (black squares) based on experimentally determined atomic positions of iron and oxygen atoms and the valence bond rule or ab initio calculation for models with iron in high spin (solid circles) and low spin (open circles) states. The curves serve as guides to the eyes. Note that according to $a b$ initio calculations in the HS state the symmetrization of the $\mathrm{H}$ bonds should be observed only at pressures above $100 \mathrm{GPa}$, but in the LS state it happens at $\sim 50 \mathrm{GPa}$.

volume drop with no change in symmetry group is usually characteristic of an electronic transition. To further elucidate the origin of the volume contraction ${ }^{57} \mathrm{Fe}$ Mössbauer spectroscopy studies were carried out in the 0-75 GPa range and in the 6-300 $\mathrm{K}$ temperature range.

The Mössbauer spectrum at ambient pressure can be well fitted with a single sextet component, with a hyperfine field $\left(H_{\mathrm{hf}}\right)$ of $50.2 \mathrm{~T}$, typical of a six-coordinated $\mathrm{Fe}^{3+}-\mathrm{O}$ species in the high-spin configuration $(S=5 / 2)$ [24]. Up to $\sim 50 \mathrm{GPa}$ the antiferromagnetic low-temperature spectra barely change. The isomer shift (IS), which is negatively proportional to the $s$ density $\left(\rho_{s}\right)$ at the Fe nucleus, decreases monotonically with pressure increase (Fig. 2, inset). Above $50 \mathrm{GPa}$ a new magnetic component appears (Fig. 4) and eventually becomes dominant at higher pressure. This component is characterized by smaller values of 


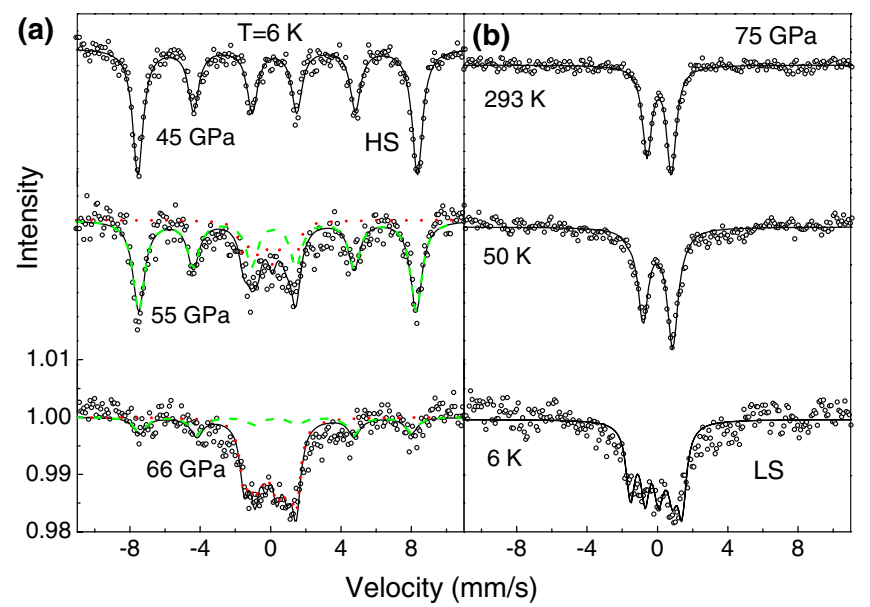

FIG. 4 (color online). Mössbauer spectra of $\mathrm{FeOOH}$ as function of pressure at $6 \mathrm{~K}$ (a) and at different temperatures at $75 \mathrm{GPa}$ (b). At $45 \mathrm{GPa}$ the spectrum (a) can still be well fitted with a single sextet. At $55 \mathrm{GPa}$ appears a new component (red dotted line), which is characterized by the significantly reduced $H_{h f}$ of $\sim 7.2 \mathrm{~T}$. This component becomes the only remaining component at $75 \mathrm{GPa}$. The quadruple splitting (QS) value barely changes upon cooling at $75 \mathrm{GPa}$ (b) and remains $\sim 2.5 \mathrm{~mm} / \mathrm{s}$. At $50 \mathrm{~K}$ a slight broadening of the doublet takes place, followed by the onset of a magnetic splitting clearly observed at $6 \mathrm{~K}$. This means a considerable drop of Neel temperature $\left(T_{N}\right)$ as compared to $T_{N}$ above $300 \mathrm{~K}$ of the low-pressure phase. Thus, the high pressure phase is characterized by the significantly reduced isomer shift and hyperfine field, lower $T_{N}$, and enlarged QS values as compared to the low pressure phase: all these changes are features of the low-spin state of $\mathrm{Fe}^{3+}$.

IS, $H_{\mathrm{hf}}(7.2 \mathrm{~T})$, Neel temperature $\left(T_{N}\right)$, and larger quadrupole splitting (QS), all typical of a $\mathrm{Fe}^{3+}$ low spin state $\left(S=1 / 2,{ }^{2} T_{2 g}\right)$. At $75 \mathrm{GPa}$ the only remaining component is this high pressure component (Fig. 4).

Additional evidence of the strong electronic transformation taking place around $45 \mathrm{GPa}$ is the sharp decrease of the $R(P, 300 \mathrm{~K})$ by 5 orders of magnitude (Ref. [22], Fig. S3). From the $T$ dependence (see inset) no metallization takes place up to the highest $P$. Thus we can conclude that around $45 \mathrm{GPa}$ a corroborating electronic and isostructural transformation takes place, namely, a HS-LS transition leading to a significant reduction of the $\mathrm{Fe}^{3+}$ ionic radius and the corresponding large drop in volume.

While the quality of the single crystal deteriorates upon the transition, we found the x-ray data still sufficient for accurate determination of the positions of iron and oxygen atoms in the high pressure phase (Ref. [22], Table S1). This phase is characterized by significantly shorter $\mathrm{O} 1 \cdots \mathrm{O} 2$ distances, the same as measured in ice- $X$ at $145 \mathrm{GPa}$ where symmetrization of the hydrogen bond has been observed [16] (Fig. 2). Furthermore, the distortion of the $\mathrm{Fe}(\mathrm{O} \cdots \mathrm{H})_{3}(\mathrm{O}-\mathrm{H})_{3}$ moiety is strikingly reduced (Figs. 1 and 3) with the $\mathrm{O}_{a}-\mathrm{Fe}-\mathrm{O} 2_{a}$ angle approaching $180^{\circ}$ and nearly equal $\langle\mathrm{Fe}-\mathrm{O} 2\rangle$ and $\langle\mathrm{Fe}-\mathrm{O} 1\rangle$ bond lengths. The exact position of the hydrogen atom cannot be directly determined from XRD data, but the interatomic distance of the hydroxyl and the $\mathrm{O} \cdots \mathrm{H}$ species can be derived using the valence bond rule and the experimentally measured atomic positions of iron and oxygen atoms (for details see the Supplemental Material [22], and references therein). As can be seen at $P \geq 50 \mathrm{GPa}$ [Fig. 3(a)] the difference $D$ between the interatomic distances of $(\mathrm{O} 1-\mathrm{H})$ and $(\mathrm{H} \cdots \mathrm{O} 2)$ vanishes. Thus, we conclude, that the single crystal XRD data provide strong evidence for a symmetric hydrogen bond in the high-pressure $\mathrm{FeOOH}$ phase. This H-bonds symmetrization is induced by the spin crossover, which converts the largely asymmetric polyhedra of $\mathrm{Fe}_{\mathrm{HS}}^{3+}(\mathrm{O}-\mathrm{H})_{3}(\mathrm{O} \ldots \mathrm{H})_{3}$ into an axially symmetric $\mathrm{Fe}_{\mathrm{LS}}^{3+}(\mathrm{O}-\mathrm{H})_{6}$ orthorhombic octahedra, accompanied by a $\sim 11 \%$ volume collapse.

It is worth mentioning that solely a reduction of the $\mathrm{Fe}^{3+}$ ionic radius, due to the transition to the LS state, is not sufficient to fully explain the obtained result. In diaspore $(\alpha-\mathrm{AlOOH})$, an isostructural oxyhydroxide with much smaller molar volume than $\alpha-\mathrm{FeOOH}$ and smaller $\mathrm{Al}^{3+}$ ionic radius even compared with the low-spin $\mathrm{Fe}^{3+}$, no hydrogen bond symmetrization has been observed to 50 $\mathrm{GPa}$ and it has been theoretically predicted to take place only at $\sim 110 \mathrm{GPa}[21,24,25]$. Importantly, the observed drastic volume change in $\alpha-\mathrm{FeOOH}$ at the spin transition is a consequence not only of the $\mathrm{Fe}^{3+}-\mathrm{O}$ bond length reduction, but also of the change of the $\mathrm{O} 1 \cdots \mathrm{O} 2$ distances which are reduced by $\sim 10 \%$. The latter reflects, obviously, a drastic redistribution of the electron density in the $\mathrm{Fe}(\mathrm{O} \cdots \mathrm{H})_{3}(\mathrm{O}-\mathrm{H})_{3}$ moiety following the spin transition.

To further substantiate our findings we employed $a b$ initio calculations in order to investigate the stability and structural properties of $\mathrm{HS}$ and $\mathrm{LS} \mathrm{Fe}^{3+}$ states in $\alpha$-FeOOH (Ref. [22], Fig. S4). The antiferromagnetic (AFM) HS state with a local magnetic moment $\mu\left(\mathrm{Fe}^{3+}\right) \sim$ $4.2 \mu_{B}$ was found to have the lowest total energy, i.e., to be the most stable configuration in the $0-57 \mathrm{GPa}$ range (Ref. [22], Fig. S4). Above $57 \mathrm{GPa}$ (inset Fig. S4) the stable configuration becomes the LS phase with $\mu \sim$ $1.1 \mu_{B}$, in excellent agreement with the present experimental results. The theoretical analysis even implies [Fig. 3(b)] that the spin crossover results in nearly symmetric hydrogen bonds. Thus the two phenomena-electronic transition in $\mathrm{Fe}^{3+}$ and modification of the hydrogen bond, resulting in the dissociation of the hydroxyl, are closely interlinked.

Our experimental studies combined with the ab initio calculations suggest that hydrogen bond symmetrization may occur in other inorganic oxy-hydroxide transition metal (TM) species at relatively low pressures in cases of pressure-induced electronic processes, such as spin crossover or pressure-induced oxidation of the TM ion which eventually leads to substantial volume reduction and change of electronic state. Such an effect may be common for crystalline materials and minerals containing water and 
transition metals, particularly for components of Earth and planetary mantles. Indeed, water is expected to be carried into Earth's interiors by ferric iron bearing oxides and silicates [26] and, induced by spin transition in iron, at conditions of the middle lower mantle, changes in hydrogen bonding may significantly affect water balance and dynamics.

Support from the German Science Foundation (DFG), German Ministry for Education and Research, the Swedish Research Council (VR) Projects No. 621-2011-4426 and 2011- 42-59, LiLi-NFM, and the Swedish Foundation for Strategic Research (SSF) program SRL10-0026 is gratefully acknowledged. S. I. S. acknowledges support from the Swedish Government Strategic Research Area Grant in Materials Science to AFM research environment at LiU. Supercomputer resources were provided by the Swedish National Infrastructure for Computing (SNIC).

*leonid.dubrovinsky@uni-bayreuth.de

[1] E. Arunan et al., Pure Appl. Chem. 83, 1637 (2011).

[2] W. B. Holzapfel, J. Chem. Phys. 56, 712 (1972).

[3] M. Benoit, D. Marx, and M. Parrinello, Nature (London) 392, 258 (1998)

[4] L. Lin, J. A. Morrone, and R. Car, J. Stat. Phys. 145, 365 (2011).

[5] G. Gilli and P. Gilli, The Nature of the Hydrogen Bond (Oxford University Press, New York, 2009).

[6] H. Yang, R. Lu, R. T. Downs, and G. Costin, Acta Crystallogr. Sect. E 62, i250 (2006).

[7] K. Aoki, E. Katoh, H. Yamawaki, M. Sakashita, and H. Fujihisa, Physica (Amsterdam) B 265, 83 (1999).

[8] A. Sano-Furukawa, K. Komatsu, C. B. Vanpeteghem, and E. Ohtani, Am. Mineral. 93, 1558 (2008).

[9] A. Sano-Furukawa, H. Kagi, T. Nagai, S. Nakano, S. Fukura, D. Ushijima, R. Iizuka, E. Ohtani, and T. Yagi, Am. Mineral. 94, 1255 (2009).
[10] A. Hushur, M. H. Manghnani, J. R. Smyth, Q. Williams, E. Hellebrand, D. Lonappan, Y. Ye, P. Dera, and D. J. Frost, J. Geophys. Res. 116, 1 (2011).

[11] M. Matsui, K. Komatsu, E. Ikeda, A. Sano-Furukawa, H. Gotou, and T. Yagi, Am. Mineral. 96, 854 (2011).

[12] A. Goncharov, M. Manaa, J. Zaug, R. Gee, L. Fried, and W. Montgomery, Phys. Rev. Lett. 94, 065505 (2005).

[13] P. Pruzan, J. Mol. Struct. 322, 279 (1994).

[14] A. F. Goncharov, V. V. Struzhkin, M. S. Somayazulu, R. J. Hemley, and H.K. Mao, Science 273, 218 (1996).

[15] K. Aoki, H. Yamawaki, M. Sakashita, and H. Fujihisa, Phys. Rev. B 54, 15673 (1996).

[16] P. Loubeyre, R. LeToullec, E. Wolanin, M. Hanfland, and D. Hausermann, Nature (London) 397, 503 (1999).

[17] L. Dubrovinsky et al., High Press. Res. 30, 620 (2010).

[18] T. Nagai, H. Kagi, and T. Yamanaka, Am. Mineral. 88, 1423 (2003).

[19] A. E. Gleason, R. Jeanloz, and M. Kunz, Am. Mineral. 93, 1882 (2008).

[20] K. Otte, R. Pentcheva, W. W. Schmahl, and J. R. Rustad, Phys. Rev. B 80, 205116 (2009).

[21] D. Tunega, J. Phys. Chem. C 116, 6703 (2012).

[22] See Supplemental Material at http://link.aps.org/ supplemental/10.1103/PhysRevLett.111.175501 for additional tables, figures, descriptions of methods, and discussion.

[23] A. Friedrich, D. J. Wilson, E. Haussühl, B. Winkler, W. Morgenroth, K. Refson, and V. Milman, Phys. Chem. Miner. 34, 145 (2007).

[24] S. Bocquet, R. J. Pollard, and J. D. Cashion, Phys. Rev. B 46, 11657 (1992).

[25] A. Friedrich, E. Haussuhl, R. Boehler, W. Morgenroth, E. A. Juarez-Arellano, and B. Winkler, Am. Mineral. 92, 1640 (2007).

[26] A. H. Peslier, A. B. Woodland, D. R. Bell, and M. Lazarov, Nature (London) 467, 78 (2010). 